\title{
Numerical study on the correlation between CP violation in neutrino oscillations and baryogenesis
}

\author{
T. Endoh, T. Morozumi and A. Purwanto* \\ Department of Physics, Hiroshima University, Higashi Hiroshima - 739-8526, Japan
}

\begin{abstract}
We numerically study the correlation between $\mathrm{CP}$ violation in the neutrino oscillations and baryogenesis in the seesaw model. In this study we get the heavy Majorana neutrino masses and lepton number asymmetries from their decays by fitting the data of neutrino oscillations and by working on some hypothesis of the Dirac-Yukawa term for neutrinos.
\end{abstract}

\section{Introduction}

In the previous paper[1] we have identified the $\mathrm{CP}$ violating phases of the seesaw model and found six independent phases. In the case where the heavy right-handed Majorana mass has a strong hierarchy in the diagonal basis $M_{1} \gg M_{2} \gg M_{3}$, the diagonalization of the seesaw matrix is possible under the assumption that the elements in Yukawa-Dirac mass matrix are rather universal. Among six phases, three of them are identified as a Dirac phase and two Majorana phases in the light neutrino sector while the remaining three arise from the mixing of the light neutrinos and heavy neutrinos. Other result is that three phases of the low energy sector in principle can be determined by using neutrino oscillation and double beta decay experiments. The remaining three appears in the lepton number asymmetry of heavy Majorana neutrinos decays. Under the assumption, it was shown the phases in low energy sector and the phases in high energy sector are not related each others and the correlation between $\mathrm{CP}$ violation at low energy and $\mathrm{CP}$ violation at high energy is rather weak.

In this paper, we explore more general case. Following the arguments given by [2], we assume that the Yukawa-Dirac mass term is rather hierarchical and their magnitudes are given by up type quark masses. By reducing numbers of independent parameters, we can study the case that the correlation does exist. The purpose of this paper

* On leave from Jurusan Fisika FMIPA-ITS Surabaya, and Universitas Muhammadiyah Malang Indonesia. is to study numerically the correlation between $\mathrm{CP}$ violating phases in MNS matrix and $\mathrm{CP}$ violating phases for baryogenesis through leptogenesis.

\section{Seesaw Model and MNS Matrix}

The mass Lagrangian of the seesaw model has the form

$\mathcal{L}_{m}=-\frac{1}{2}\left(\nu_{L}, N_{R}^{c}\right) M_{\nu}\left(\begin{array}{c}\nu_{L}^{c} \\ N_{R}\end{array}\right)+$ h.c.,

where $M_{\nu}$ is $6 \times 6$ mass matrix

$M_{\nu}=\left(\begin{array}{cc}0 & m_{D} \\ m_{D}^{T} & M\end{array}\right)$,

where $m_{D}, M$ are Dirac and Majorana mass matrix respectively. This $M_{\nu}$ can be diagonalized by unitary matrix $V$, i.e., $m_{d}=V^{T} M_{\nu} V$, where $V$ is given as $\left(\nu_{L} N_{R}^{c}\right)^{T}=V^{*} \nu_{L}^{\alpha}$.

In the non-decoupling case [1] and by using the mass eigenstate for charged lepton, the $V_{M N S}$ matrix is the submatrix $3 \times 6$ of $V$ itself. General analysis on this extended $3 \times 6$ MNS matrix results in six independent $\mathrm{CP}$ phases.

Rewriting $m_{d}$ in the form $\operatorname{diag}\left(m_{\nu}, M_{d}\right), V$ in the submatrices $\mathrm{K}, \mathrm{R}, \mathrm{S}, \mathrm{T}$, and the order of $\mathrm{S}$ and $\mathrm{R}$ as given in[3], then the diagonalization we will obtain a usual relation of the seesaw model

$m_{\nu}=-K^{\dagger} m_{D} \frac{1}{M} m_{D}^{T} K^{*}$.

Further analysis gives the charged current which shows that $K$ and $R$ give the charged current couplings of charged leptons to the light neu- 
trinos $\nu_{L}$ and to the heavy neutrinos $N_{R}$, respectively. In the decoupling limit, $R$ can be neglected and only $3 \times 3$ MNS matrix $K$ is relevant.

\section{Correlation between the Phases}

We consider the diagonalization of Eq.(3), and for calculational purpose we take all six phases of $3 \times 3$ MNS matrix appear. We parameterize

$m_{D}=U Y_{\triangle}$,

where $U$ is the unitary matrix and $Y_{\triangle}$ is triangle matrix whose real diagonal elements $y_{i}$ and complex off-diagonal elements $y_{i j}$ where $i>j$. To make a connection between MNS matrix and lepton number asymmetry we set $U=1$ and suppress six independent parameters. With this working hypothesis, from the low energy neutrino oscillation data, we can determine the scales of heavy Majorana neutrinos and the lepton number asymmetries from their decays.

Without loose of generality we take matrix $M$ is real diagonal, $M=\operatorname{diag}\left(M_{1}, M_{2}, M_{3}\right)$. Expansion Eq.(3) in component $\left(K m_{\nu} K^{T}\right)_{i j}=$ $\left(-Y_{\triangle} \frac{1}{M} Y_{\triangle}^{T}\right)_{i j}$, we have

$$
\begin{aligned}
M_{1} & =\frac{1}{m_{11}} y_{1}^{2}, \\
M_{2} & =\frac{m_{11}}{m_{11} m_{22}-m_{12}^{2}} y_{2}^{2}, \\
M_{3} & =\frac{m_{11} m_{22}-m_{12}^{2}}{\operatorname{det} \mid K m_{\nu} K^{T}} y_{3}^{2},
\end{aligned}
$$

and

$$
\begin{aligned}
y_{21} & =\frac{m_{12}}{m_{11}} y_{1}, \\
y_{31} & =\frac{m_{13}}{m_{11}} y_{1}, \\
y_{32} & =\frac{m_{11} m_{23}-m_{12} m_{13}}{m_{11} m_{22}-m_{12}^{2}} y_{2},
\end{aligned}
$$

where $m_{i j}=\left(K m_{\nu} K^{T}\right)_{i j}$. The Eq.(5) leads to three constraints for six phases of general unitary MNS matrix, hence the MNS matrix has only three free phases as we demand. Whereas the Eq.(6) gives relation between the phases in low and high energy sector.

Because both $M_{i}$ and $y_{i}$ are real then we can easily see that Eq.(5) gives simple con- straints i.e. each denominator of these equations must be real. By using the standard parameterization of the CKM matrix [4], we parameterize the MNS matrix $K$ of the form $K=$ $\operatorname{diag}\left(e^{i \beta_{1}}, e^{i \beta_{2}}, e^{i \beta_{3}}\right) V_{C K M}(\delta) \operatorname{diag}\left(1, e^{i \alpha_{1}}, e^{i \alpha_{2}}\right)$. These $K, m_{\nu}$ and the above constraints yield $\beta$ as function of neutrino masses $m_{i}$, mixing angles $\theta_{i j}$ and $\mathrm{CP}$ phases $\delta, \alpha_{1}, \alpha_{2}$. These $\beta_{i}$ are not independent phases and can be absorbed by charged lepton fields, and only Dirac phase $\delta$ and majorana phases $\alpha_{1}, \alpha_{2}$ are independent phases. Next, the reality condition of $M$ will give $M_{i}=M_{i}\left(y_{i}, m_{1}, m_{2}, m_{3}, \theta_{12}, \theta_{13}, \theta_{23}, \delta, \alpha_{1}, \alpha_{2}\right)$ and therefore, it is possible to estimate their values using the neutrino data on neutrino masses, mixing angles and some assumption on the lightest $m_{1}$ and diagonal elements $y_{i}$. For numerical calculation, we will set $y_{1}=O\left(m_{u}\right), y_{2}=O\left(m_{c}\right)$ and $y_{3}=O\left(m_{t}\right)$.

To determine the correlation between the phases in high energy sector live in $y_{i j}$ and the phases in low energy sector i.e, $\delta, \alpha_{1}$, and $\alpha_{2}$ we factor out the phases from $y_{i j}$ as $\left|y_{i j}\right| e^{i f_{i j}}$. This factorization and taking into account the reality of $y_{i}$ as well as the previous constraints also give the simple constraints i.e. multiplication $\exp \left(-i f_{i j}\right)$ to each numerator of $y_{i j}$ in Eq.(6) must be real.

\section{Numerical Results}

Take the values of neutrino masses $m_{3}=$ $5.5 \times 10^{-2} \mathrm{eV}, \quad m_{2}=4.5 \times 10^{-3} \mathrm{eV}$, and $m_{1}=$ $10^{-2} \times m_{2}$ and mid values of mixing angles [5] with all phases are zero

$K=\left(\begin{array}{ccc}0.80643 & 0.585905 & 0.0799147 \\ -0.474852 & 0.561102 & 0.677997 \\ 0.352402 & -0.584705 & 0.730707\end{array}\right)$.

These values are combined with $y_{1}=$ $0.003 \mathrm{GeV}, y_{2}=1.25 \mathrm{GeV}, y_{3}=170 \mathrm{GeV}$. After substituting them into Eq.(5), we get the masses of right-handed neutrino,

$M_{1}=4.67 \times 10^{6} \mathrm{GeV}$,
$M_{2}=9.49 \times 10^{10} \mathrm{GeV}$,
$M_{3}=8.22 \times 10^{16} \mathrm{GeV}$. 


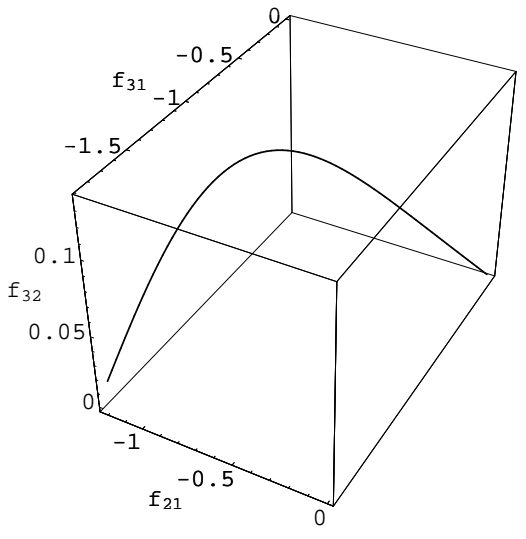

Figure 1. The correlation between $f_{i j}$ and $\delta$. We set $\alpha_{i}=0$.

An example of correlation between $\mathrm{CP}$ phases in low energy sector $(\delta)$ and CP phases for leptogenesis $\left(f_{21}, f_{31}, f_{32}\right)$ is shown in Figure 1 . We change $\delta$ from 0 to $\pi$.

Using the above results we evaluate the lepton number asymmetry coming from the decay of massive right-handed Majorana neutrino $N_{i}$ into charged leptons $l$ and Higgs fields $\chi$

$$
\begin{aligned}
a_{s}\left(N_{i} \rightarrow l^{\mp} \chi^{ \pm}\right) & =\frac{v^{-2}}{4 \pi\left(y_{\triangle}^{\dagger} y_{\triangle}\right)^{i i}} \sum_{k} \operatorname{Im}\left[\left(y_{\triangle}^{\dagger} y_{\triangle}\right)^{i k}\right]^{2} \\
& \times\left[\mathrm{I}\left(\frac{M_{k}{ }^{2}}{M_{i}{ }^{2}}\right)+\frac{M_{i}^{2}}{M_{i}{ }^{2}-M_{k}{ }^{2}}\right] \cdot(9)
\end{aligned}
$$

where $v=246 \mathrm{GeV}$. The dependence of asymmetry on low $\mathrm{CP}$ phases can be obtained, and the dependence on Dirac phase $\delta$ is given by Figure 2 . From this figure, we can see the order of asymmetry from $N_{R 1}$ is $10^{-10}$, and the asymmetry of the heavier ones are larger.

\section{Conclusions}

In the present paper, we study the correlation between $\mathrm{CP}$ violation at low energy and at high energy. Under the working hypothesis on the Dirac mass term of neutrino sector, we can de-

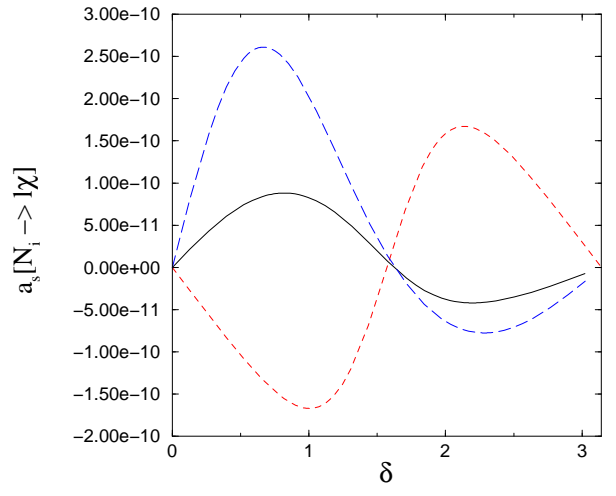

Figure 2. The asymmetries $a_{s}$ as function of $\delta$. Dashed, long-dashed and solid line are $a_{s 1}, a_{s 2} / 10$ and $a_{s 3} / 10^{4}$ respectively. We set $\alpha_{i}=0$.

termine $\mathrm{CP}$ violating phases which are related to baryogenesis through leptogenesis from the three $\mathrm{CP}$ violating phases in MNS matrix, the neutrinos mixing angles and their mass differences. The correlation between the lepton number asymmetry and the $\mathrm{CP}$ violating phases in MNS matrix is numerically studied. We estimate the masses and the decay widths of three heavy Majorana neutrinos. These are sufficient for prediction of the baryon number asymmetry.

\section{Acknowledgment}

We would like to thank organizers of KEKTC5. This work is supported by the Grand-in-Aid for scientific research No.13640290 from the Ministry of Education; Science and Culture of Japan.

\section{REFERENCES}

1. T. Endoh, T. Morozumi, T.Onogi and A. Purwanto, Phys. Rev. D 64 013006(2001), Erratum-ibid. D 64 059904(2001).

2. G.C.Branco, T.Morozumi, B.M. Nobre and M.N.Rebelo, Nucl. Phys. B617, 475 (2001)

3. J.Hashida, T. Morozumi and A. Purwanto, Prog. Theor. Phys. 103379 (2000).

4. L. Chau and W. Keung, Phys. Rev. Lett. 53, 1802 (1984). 
5. M. Fukugita and M. Tanimoto, Phys. Lett. B515, 30 (2001). 